Pure and Applied Mathematics Quarterly

Volume 3, Number 3

(Special Issue: In honor of

Leon Simon, Part 2 of 2$)$

$827-840,2007$

\title{
Isometric Embedding of Negatively Curved Disks in the Minkowski Space
}

\author{
Bo Guan \\ Dedicated to Professor Leon Simon on his 60th birthday
}

\section{INTRODUCTION}

The hyperbolic plane $\mathbb{H}^{2}$ has a canonical isometric embedding in the Minkowski space $\mathbb{R}^{2,1}$ given by the hyperboloid

$$
x_{3}=\sqrt{1+\left|x^{\prime}\right|^{2}}, \quad x^{\prime} \in \mathbb{R}^{2} .
$$

It seems an interesting question whether a two-dimensional simply connected complete Riemannian manifold $(M, g)$ of negative curvature always admits an isometric embedding in $\mathbb{R}^{2,1}$. This is equivalent (see Section 2) to solving the Monge-Ampère type equation on $(M, g)$ :

$$
\operatorname{det} \nabla^{2} u=-K_{g}\left(1+|\nabla u|^{2}\right) \operatorname{det} g
$$

where $K_{g}$ is the (intrinsic) curvature of $g$. Note that this equation is elliptic when $K_{g}<0$. One can also ask other questions concerning local or global isometric embedding in $\mathbb{R}^{2,1}$ for two-dimensional Riemannian manifolds. In this note we shall consider the problem for compact disks with negative curvature.

Received February 28, 2006 .

Research of the author was supported in part by an NSF grant. 
Theorem 1.1. Let $g$ be a smooth metric of negative curvature on a compact 2-disk $\mathcal{D}$ with smooth boundary $\partial \mathcal{D}$. Suppose $\partial \mathcal{D}$ has positive geodesic curvature. Then there exists a smooth isometric embedding $\mathbf{x}:(\mathcal{D}, g) \rightarrow \mathbb{R}^{2,1}$ with $\mathbf{x}(\partial \mathcal{D}) \subset$ $\left\{x_{3}=0\right\}$.

This can be viewed as a counterpart to a theorem of Pogorelov [23] and Hong [14], which states that a positively curved 2-disk with positive geodesic curvature along its boundary admits an isometric embedding in $\mathbb{R}^{3}$ with planar boundary.

Without the assumption of positive geodesic curvature along $\partial \mathcal{D}$ we shall prove a weaker existence result which seems to have no counterpart in the positive curvature case.

Theorem 1.2. Let $(\mathcal{D}, g)$ be a smooth compact disk of negative curvature with smooth boundary $\partial \mathcal{D}$. Suppose $(\mathcal{D}, g)$ is geodesically star-shaped with respect to an interior point in $\mathcal{D}$. Then $(\mathcal{D}, g)$ admits a smooth isometric embedding into $\mathbb{R}^{2,1}$.

We say $(\mathcal{D}, g)$ is geodesically star-shaped with respect to $x_{0} \in \mathcal{D}$ if the exponential map $\exp _{x_{0}}$ is a diffeomorphism from a star-shaped domain (with respect to the origin) in the tangent plane $T_{x_{0}} \mathcal{D}$ onto $\mathcal{D}$.

Problems concerning isometric embedding of surfaces in the Euclidean space $\mathbb{R}^{3}$ have received much attention. In 1906 Weyl considered the problem whether a smooth metric on $\mathbb{S}^{2}$ with positive curvature always admits an isometric embedding in $\mathbb{R}^{3}$. This problem, known as the Weyl problem, was studied subsequently by Lewy, Alexanderov, and finally solved by Nirenberg [19] and Pogorelov [22] independently; their results were extended by Guan-Li [8] and Hong-Zuily [18] to the nonnegative curvature case. In [25] Yau posed the question of finding isometric embedding with prescribed boundary or with boundary contained in a given surface in $\mathbb{R}^{3}$ for compact disks of positive curvature. Important contributions to the area were made by Pogorelov [23] and Hong [12]-[17] who established remarkable existence results for compact disks of positive curvature, and for complete noncompact surfaces of nonnegative curvature. Hong [14] also considered the problem for complete noncompact surfaces of negative curvature. We should also mention the breakthroughs of Lin [20], [21], and the more recent work of HanHong-Lin [11] and Han [9], [10] for local isometric embedding in $\mathbb{R}^{3}$. It would be 
interesting to study corresponding questions for isometric embedding in $\mathbb{R}^{2,1}$ of negatively or nonpositively curved surfaces.

We shall derive equation (1.2) in Section 2 and show the equivalence of its solvability to finding isometric embedding in $\mathbb{R}^{2,1}$ of a surface, therefore reducing the proof of Theorems 1.1 and 1.2 to the Dirichlet problems for (1.2). In Section 3 we construct subsolutions for (1.2) when $K_{g}<0$, which implies the existence of solutions according to the general theory of Monge-Ampère equations. We shall consider more general equations and boundary data in higher dimensions.

\section{Basic Formulas}

In this section we derive equation (1.2) for isometric embedding of a surface into $\mathbb{R}^{2,1}$. We begin with some basic notation and formulas.

Let $\mathbb{R}^{n, 1}(n \geq 2)$ be the $(n+1)$-dimensional Minkowski space which is $\mathbb{R}^{n+1}$ equipped with the Lorentz metric

$$
d s^{2}=\sum_{i=1}^{n} d x_{i}^{2}-d x_{n+1}^{2} .
$$

We use $\langle\cdot, \cdot\rangle$ to denote the Lorentz pairing, i.e.

$$
\langle v, w\rangle=\sum_{i=1}^{n} v_{i} w_{i}-v_{n+1} w_{n+1} .
$$

A hypersurface $\Sigma$ in $\mathbb{R}^{n, 1}$ is spacelike if $d s^{2}$ induces a Riemannian metric on $\Sigma$, that is, the restriction of the Lorentz pairing to the tangent plane of $\Sigma$ at any point is positive definite.

Let $\Sigma^{n}$ be a spacelike hypersurface in $\mathbb{R}^{n, 1}$. We shall use $\nabla$ and $\Delta$ to denote the Levi-Civita connection and the Laplace-Beltrami operator on $\Sigma$, respectively, while $D$ the standard connection of $\mathbb{R}^{n, 1}$. Let $\nu$ be the timelike unit normal of $\Sigma$, i.e.

$$
\langle\nu, \nu\rangle=-1 \text {. }
$$

Let $e_{1}, \ldots, e_{n}$ be a local orthonormal frame on $\Sigma$. The second fundamental form of $\Sigma$ is defined as

$$
h_{i j}=\left\langle D_{e_{i}} \nu, e_{j}\right\rangle, \quad 1 \leq i, j \leq n .
$$


We have $h_{i j}=h_{j i}$,

$$
D_{e_{i}} \nu=h_{i j} e_{j}, \quad D_{e_{i}} e_{j}=h_{i j} \nu+\Gamma_{i j}^{k} e_{k},
$$

where $\Gamma_{i j}^{k}=\left\langle\nabla_{e_{i}} e_{j}, e_{k}\right\rangle$ are the Christoffel symbols, and the Codazzi equation

$$
\nabla_{k} h_{i j}=\nabla_{i} h_{j k} .
$$

The Riemannian curvature tensor is given by the Gauss equation (see e.g. [4])

$$
R_{i j k l}=-h_{i k} h_{j l}+h_{i l} h_{j k} .
$$

The mean and Gauss curvatures of $\Sigma$ are

$$
H=\frac{1}{n} \sum h_{i i}, \quad K=\operatorname{det} h_{i j}
$$

respectively, while the norm of the second fundamental form is given by

$$
|A|^{2}=\sum h_{i j}^{2}
$$

Thus for a spacelike surface in $\mathbb{R}^{2,1}$ its intrinsic curvature is $R_{1212}=-K$.

Let $\mathbf{x}$ be the position vector of $\Sigma$ in $\mathbb{R}^{n, 1}$ and define

$$
u=-\langle\mathbf{x}, \mathbf{e}\rangle, \eta=\langle\mathbf{x}, \nu\rangle, \quad z=\langle\mathbf{x}, \mathbf{x}\rangle
$$

which are called the height, support, and extrinsic distance functions of $\Sigma$, respectively. Here $\mathbf{e}=(0, \ldots, 0,1) \in \mathbb{R}^{n, 1}$ is the unit vector in the $x_{n+1}$ (time) direction:

$$
\langle\mathbf{e}, \mathbf{e}\rangle=-1
$$

We have $D_{e_{i}} \mathbf{x}=e_{i}$,

$$
\nabla_{i j} \mathbf{x}:=D_{e_{i}} D_{e_{j}} \mathbf{x}-\Gamma_{i j}^{k} D_{e_{k}} \mathbf{x}=h_{i j} \nu
$$

Thus

$$
\begin{gathered}
\nabla_{i} u=-\left\langle e_{i}, \mathbf{e}\right\rangle, \\
\nabla_{i j} u=-\langle\nu, \mathbf{e}\rangle h_{i j},
\end{gathered}
$$

and

$$
|\nabla u|^{2}=\sum_{i=1}^{n}\left\langle e_{i}, \mathbf{e}\right\rangle^{2}=\langle\mathbf{e}, \mathbf{e}\rangle+\langle\nu, \mathbf{e}\rangle^{2}=-1+\langle\nu, \mathbf{e}\rangle^{2} .
$$

Consequently, $u$ satisfies the Monge-Ampère type equation

$$
\operatorname{det} \nabla_{i j} u=K\left(1+|\nabla u|^{2}\right)^{\frac{n}{2}} \text {. }
$$


Let $(\mathcal{D}, g)$ be a two-dimensional Riemannian manifold and $\mathbf{x}:(\mathcal{D}, g) \rightarrow \Sigma \subset$ $\mathbb{R}^{2,1}$ an isometric embedding. Thus $\Sigma$ is naturally spacelike in $\mathbb{R}^{2,1}$ and we see that the function $u:=\langle\mathbf{x}, \mathbf{e}\rangle$ satisfies equation (1.2) in $\mathcal{D}$. Conversely, a solution of $(1.2)$ in $\mathcal{D}$ yields an isometric embedding of $(\mathcal{D}, g)$ into $\mathbb{R}^{2,1}$. This is a consequence of the following fact.

Lemma 2.1. For $u \in C^{2}(\mathcal{D}, g)$ the Gauss curvature of the metric $g_{1}=g+d u^{2}$ is

$$
K_{g_{1}}=\frac{1}{1+|\nabla u|^{2}}\left(K_{g}+\frac{\operatorname{det} \nabla^{2} u}{\left(1+|\nabla u|^{2}\right) \operatorname{det} g}\right) .
$$

In particular, $K_{g_{1}}=0$ if $u$ is a solution of (1.2).

This lemma guarantees that for a smooth solution $u$ of (1.2) we can always find a smooth isometry from $\left(\mathcal{D}, g+d u^{2}\right)$ into $\mathbb{R}^{2}$ when $\mathcal{D}$ is simply connected since $g+d u^{2}$ is flat. If we use $(x, y): \mathcal{D} \rightarrow \mathbb{R}^{2}$ to denote this isometry then the desired isometric embedding $\mathbf{x}:(\mathcal{D}, g) \rightarrow \Sigma \subset \mathbb{R}^{2,1}$ is given by $\mathbf{x}=(x, y, u)$.

For the proof of Lemma 2.1 one can follow, for example, that of Lemma 1 in [16] with slight modifications. So we omit it here.

In the rest of this section we derive equations for geometric quantities of $(\mathcal{D}, g)$. Let $\mathcal{L}$ be the linear operator defined as

$$
\mathcal{L} v=h^{i j} \nabla_{i j} v, \quad v \in C^{2}(\mathcal{D}) .
$$

First, differentiating $K=\operatorname{det} h_{i j}$ we obtain

$$
h^{i j} h_{i j k}=(\log K)_{k}
$$

and

$$
h^{i j} h_{i j k k}-h^{i b} h^{a j} h_{i j k} h_{a b k}=(\ln K)_{k k}
$$

where $h_{i j k}=\nabla_{k} h_{i j}, h_{i j k l}=\nabla_{l k} h_{i j}$, etc. Next, we calculate

$$
\begin{aligned}
\nabla_{i j} \nu & :=D_{e_{i}} D_{e_{j}} \nu-\Gamma_{i j}^{k} D_{e_{k}} \nu \\
& =D_{e_{i}}\left(h_{j k} e_{k}\right)-\Gamma_{i j}^{k} h_{k l} e_{l} \\
& =h_{i k} h_{j k} \nu+h_{j k i} e_{k} \\
& =h_{i k} h_{j k} \nu+h_{i j k} e_{k}
\end{aligned}
$$

by the Codazzi equation, and

$$
\nabla_{i} z=2\left\langle\mathbf{x}, e_{i}\right\rangle
$$




$$
\nabla_{i j} z=2 \delta_{i j}+2 \eta h_{i j}
$$

Thus

$$
\begin{gathered}
|\nabla z|^{2}=4 \sum_{i=1}^{n}\left\langle\mathbf{x}, e_{i}\right\rangle^{2}=4 z+4 \eta^{2} \\
\mathcal{L} z=2 \sum_{i} h^{i i}+2 n \eta \\
\mathcal{L} \mathbf{x}=n \nu
\end{gathered}
$$

by $(2.5)$, and

$$
\mathcal{L} \nu=n H \nu+\nabla \ln K
$$

Therefore,

$$
\begin{aligned}
\mathcal{L} \eta & =\langle\mathbf{x}, \mathcal{L} \nu\rangle+2 h^{i j}\left\langle\nabla_{i} \mathbf{x}, \nabla_{j} \nu\right\rangle+\langle\nu, \mathcal{L} \mathbf{x}\rangle \\
& =n H \eta+n+\langle\mathbf{x}, \nabla \ln K\rangle
\end{aligned}
$$

Note that

$$
h^{i j} \eta_{i} \eta_{j}=h_{i j}\left\langle\mathbf{x}, e_{i}\right\rangle\left\langle\mathbf{x}, e_{j}\right\rangle=\frac{1}{4} h_{i j} z_{i} z_{j}
$$

So

$$
\frac{1}{4} \mathcal{L}\left(|\nabla z|^{2}\right)=\mathcal{L}\left(z+\eta^{2}\right)=2 \sum h^{i i}+4 n \eta+2 n H \eta^{2}+2 \eta\langle\mathbf{x}, \nabla \ln K\rangle+\frac{1}{2} h_{i j} z_{i} z_{j} .
$$

Finally, from

$$
h_{i j k l}-h_{i j l k}=\sum_{m} h_{i m} R_{m j k l}+\sum_{m} h_{m j} R_{m i k l} .
$$

and the Codazzi and Gauss equations, we derive

$$
\begin{aligned}
\mathcal{L} H & =\frac{1}{n} \sum_{k} h^{i j} h_{k k i j}=\frac{1}{n} \sum_{k} h^{i j} h_{i k k j} \\
& =\frac{1}{n} \sum_{k} h^{i j} h_{i k j k}+\frac{1}{n} \sum_{k, m} h^{i j} h_{i m} R_{m k k j}+\frac{1}{n} \sum_{k, m} h^{i j} h_{m k} R_{m i k j} \\
& =\frac{1}{n} \sum_{k} h^{i j} h_{i j k k}+n H^{2}-|A|^{2} \\
& =\frac{1}{n} \Delta \ln K+\frac{1}{n} \sum_{k} h^{i l} h^{m j} h_{i j k} h_{l m k}+n H^{2}-|A|^{2},
\end{aligned}
$$


and

$(2.19)$

$$
\begin{aligned}
\frac{1}{2} \mathcal{L}|A|^{2}= & \sum_{k, l} h^{i j} h_{k l} h_{k l i j}+\sum_{k, l} h^{i j} h_{k l i} h_{k l j} \\
= & \sum_{k, l} h^{i j} h_{k l} h_{i j k l}+\sum_{k, l} h^{i j} h_{k l i} h_{k l j}+\sum_{k, l, m} h^{i j} h_{k l}\left(h_{i m} R_{m k j l}+h_{m k} R_{m i j l}\right) \\
= & n H|A|^{2}+\sum_{k, l} h^{i j} h_{k l i} h_{k l j}+\sum_{k} h^{i t} h^{s j} h_{k l} h_{i j k} h_{s t l} \\
& +\sum_{k, l} h_{k l}(\ln K)_{k l}-\sum_{k, l, m} h_{k l} h_{k m} h_{m l} .
\end{aligned}
$$

The last inequality should be compared with the Calabi identity [3] (see also [4])

$$
\frac{1}{2} \Delta|A|^{2}=|A|^{4}+\sum_{i, j, k} h_{i j k}^{2}+n \sum_{i, j} h_{i j} \nabla_{i j} H-n H \sum_{i, j, k} h_{i j} h_{j k} h_{k i}
$$

\section{Construction of subsolutions}

In this section we construct subsolutions for equation (1.2) from which we conclude Theorems 1.1 and 1.2. We shall do this for a general class of Hessian equations that include (1.2). Throughout this section we assume $\left(\mathcal{D}^{n}, g\right)$ to be a compact simply connected Riemannian manifold of dimension $n(n \geq 2)$ with nonpositive sectional curvature and smooth boundary $\partial \mathcal{D}$.

Let us first consider a radially symmetric function $u(x)=u(|x|)$ in $\mathbb{R}^{n}$. A straightforward calculation shows that

$$
\operatorname{det} D^{2} u=\left(\frac{u^{\prime}}{r}\right)^{n-1} u^{\prime \prime}, \quad r=|x|,
$$

Thus the Monge-Ampère equation in $\mathbb{R}^{n}$

$$
\operatorname{det} D^{2} u=\psi(x, u, D u)
$$

takes the form

$$
\left(u^{\prime}\right)^{n-1} u^{\prime \prime}=r^{n-1} \psi\left(x, u, u^{\prime}\right)
$$

for radially symmetric functions. 
Lemma 3.1. Let $f \in C^{l}\left(\mathbb{R}_{+}\right)$be a nonnegative function defined on $\mathbb{R}_{+}:=\{r \geq$ $0\}$. Then there exists a unique convex function $\phi \in C^{l+2}\left(\mathbb{R}_{+}\right)$with $\phi(0)=0$, $\phi^{\prime}(0)=0$ and

$$
\left|\phi^{\prime}\right|^{n-1} \phi^{\prime \prime}=r^{n-1} f(r)\left(1+\left|\phi^{\prime}\right|^{n}\right), \quad \forall r>0 .
$$

Moreover, $\phi$ is strictly convex where $f>0$, and

$$
\lim _{r \rightarrow+\infty} \phi(r)=+\infty
$$

unless $f \equiv 0$ on $\mathbb{R}$.

Proof. Integrating equation (3.3), we have

$$
\log \left(1+\left(\phi^{\prime}\right)^{n}\right)=n \int_{0}^{r} r^{n-1} f(r) d r, \quad r \geq 0 .
$$

Therefore,

$$
\phi^{\prime}(r)=\left(e^{h(r)}-1\right)^{\frac{1}{n}}, \quad r \geq 0
$$

where

$$
h(r):=n \int_{0}^{r} r^{n-1} f(r) d r
$$

Integrating again,

$$
\phi(r)=\int_{0}^{r}\left(e^{h(r)}-1\right)^{\frac{1}{n}} d r, \quad \forall r \geq 0 .
$$

Finally, the convexity of $\phi$ and (3.4) follow from the fact that $\phi^{\prime}(0)=0$ and $\phi "(r)>0$ whenever $f(r)>0$.

Remark 3.2. When $f$ is constant Lemma 3.1 is a special case of Lemma 3.7 in $[7]$.

We now suppose that $(\mathcal{D}, g)$ is geodesically star-shaped with respect to $x_{0} \in \mathcal{D}$. Given any positive function $\psi \in C^{\infty}(\overline{\mathcal{D}})$, define

$$
w(x):=\phi(r(x)), \quad x \in \mathcal{D}
$$

where $\phi$ is obtained from Lemma 3.1 with $f:=A \max _{\mathcal{D}} \psi, A>0$, and $r$ is the distance function from $x_{0}$

$$
r(x):=\operatorname{dist}_{g}\left(x, x_{0}\right), \quad x \in \mathcal{D} .
$$

We calculate

$$
\nabla^{2} w=\phi^{\prime} \nabla^{2} r+\phi^{\prime \prime} d r \otimes d r
$$


Since $g$ has nonpositive curvature, by the Hessian comparison principle (see, e.g., [24]) we see that $\nabla^{2} w$ is positive definite and

$$
\operatorname{det} \nabla^{2} w \geq\left(\frac{\phi^{\prime}}{r}\right)^{n-1} \phi^{\prime \prime}=f(r)\left(1+\left|\phi^{\prime}\right|^{n}\right) \geq A \psi\left(1+|\nabla w|^{n}\right) \text { in } \mathcal{D} .
$$

Therefore $w$ is a subsolution of the following Monge-Ampère equation

$$
\operatorname{det} \nabla^{2} u=\psi\left(1+|\nabla u|^{2}\right)^{\frac{n}{2}} \operatorname{det} g \text { in } \overline{\mathcal{D}}
$$

when $A$ is chosen sufficiently large. By Theorem 5.1 of [5] we obtain a locally strictly convex solution $u \in C^{\infty}(\overline{\mathcal{D}})$ of (3.8) satisfying the Dirichlet boundary condition $u=w$ on $\partial \mathcal{D}$. In particular, for $\psi=-K_{g}$ this implies Theorem 1.2.

Using the function $w$ constructed above it is possible to solve the Dirichlet problem for equation (3.8) with arbitrary smooth boundary data when $\mathcal{D}$ is strictly convex, i.e., the second fundamental form of $\partial \mathcal{D}$ is positive definite. (When $\mathcal{D}$ is a strictly convex bounded domain in $\mathbb{R}^{n}$ this was observed by P. L. Lions; see [1].) More generally, we have the following existence result for the Hessian equation

$$
\sigma_{k}\left(\nabla^{2} u\right)=\psi(x, u)\left(1+|\nabla u|^{2}\right)^{\frac{k}{2}} \text { in } \overline{\mathcal{D}},
$$

where $\sigma_{k}\left(\nabla^{2} u\right)=\sigma_{k}\left(\lambda\left(\nabla^{2} u\right)\right)$ is the $k$-th elementary symmetric function of the eigenvalues of $\nabla^{2} u$ with respect to metric $g$.

Theorem 3.3. Let $\psi \in C^{\infty}(\overline{\mathcal{D}} \times \mathbb{R}), \psi \geq 0, \psi_{u} \geq 0$, and $\varphi \in C^{\infty}(\partial \mathcal{D})$. Suppose that $\partial \mathcal{D}$ satisfies the condition

$$
\left(\kappa_{1}, \ldots, \kappa_{n-1}\right) \in \Gamma_{k-1} \text { on } \partial \mathcal{D},
$$

where $\left(\kappa_{1}, \ldots, \kappa_{n-1}\right)$ are the principal curvatures of $\partial \mathcal{D}$. Then equation (3.9) has a unique admissible solution $u \in C^{\infty}(\overline{\mathcal{D}})$ which satisfies the boundary condition

$$
u=\varphi \text { on } \partial \mathcal{D} \text {. }
$$

Here $\Gamma_{k}$ denotes the open convex cone in $\mathbb{R}^{n}$ defined as

$$
\Gamma_{k}=\left\{\lambda \in \mathbb{R}^{n}: \sigma_{j}(\lambda)>0,1 \leq j \leq k\right\} .
$$

A function $u \in C^{2}(\mathcal{D})$ is admissible if $\lambda\left(\nabla^{2} u\right) \in \Gamma_{k}$. Equation (3.9) is elliptic at an admissible solution; see e.g. [2].

By Theorem 1.3 in [6], in order to prove Theorem 3.3 we only need to construct an admissible subsolution attaining the same boundary data. 
Lemma 3.4. Suppose $\partial \mathcal{D}$ satisfies (3.10). Then for any $\varphi \in C^{\infty}(\overline{\mathcal{D}})$ and $A>0$ there exists an admissible function $\underline{u} \in C^{\infty}(\overline{\mathcal{D}})$ with

$$
\sigma_{k}^{1 / k}\left(\nabla^{2} \underline{u}\right) \geq A\left(1+|\nabla \underline{u}|^{2}\right)^{\frac{1}{2}} \text { in } \overline{\mathcal{D}}, \quad \underline{u}=\varphi \text { on } \partial \mathcal{D} .
$$

Proof. We shall modify the constructions in [2] and [6]. For convenience we assume $\sigma_{k}$ is normalized so that

$$
\sigma_{k}(1, \ldots, 1)=1
$$

Let $d$ denote the distance to $\partial \mathcal{D}$,

$$
d(x)=\operatorname{dist}_{\mathcal{D}}(x, \partial \mathcal{D}) \text { for } x \in \mathcal{D} .
$$

We may choose $\delta_{0}>0$ sufficiently small such that $d$ is a smooth function in

$$
N_{\delta} \equiv\{x \in \overline{\mathcal{D}}: 0 \leq d \leq \delta\} \quad \forall 0<\delta \leq \delta_{0},
$$

and for each point $x \in N_{\delta_{0}}$ there is a unique point $y=y(x) \in \partial \mathcal{D}$ with

$$
d(x)=\operatorname{dist}_{\mathcal{D}}(x, y) .
$$

The eigenvalues of the Hessian of $d$ in $N_{\delta_{0}}$ are given by

$$
\lambda\left(\nabla^{2} d(x)\right)=\left(-\kappa_{1}(y(x))+O(d), \ldots,-\kappa_{n-1}(y(x))+O(d), 0\right)
$$

where $\kappa_{1}(y), \ldots, \kappa_{n-1}(y)$ are the principal curvatures of $\partial \mathcal{D}$ at $y \in \partial \mathcal{D}$.

For $t>0$ consider the function in $N_{\delta_{0}}$

$$
\eta=\frac{1}{t}\left(e^{-t d}-1\right)
$$

We have

$$
\nabla^{2} \eta=e^{-t d}\left(-\nabla^{2} d+t \nabla d \otimes \nabla d\right)
$$

and

$$
\sigma_{j}\left(\nabla^{2} \eta\right)=\left(e^{-t d}\right)^{j} \sigma_{j}\left(-\nabla^{2} d\right)+t\left(e^{-t d}\right)^{j-1} \sigma_{j-1}\left(-\nabla^{2} d\right), \quad \forall 1 \leq j \leq k .
$$

By assumption (3.10) there exists $t_{0}>0$ sufficiently large such that

$$
\nabla^{2} \eta \in \Gamma_{k} \text { and } \sigma_{k}\left(\nabla^{2} \eta\right) \geq \frac{t}{2} e^{-k t d} \text { in } N_{\delta}, \quad \forall t \geq t_{0}
$$

for $\delta>0$ sufficiently small.

On the other hand,

$$
|\nabla \eta|=e^{-t d} \leq 1 \text { in } N_{\delta}
$$


Fixing $t \geq 2(16 e A)^{k}$ and $\delta=t^{-1}$, we see that

$$
\sigma_{k}^{1 / k}\left(\nabla^{2} \eta\right) \geq 8 A(1+|\nabla \eta|) \text { in } N_{\delta} .
$$

Let $h(s)$ be a smooth convex function on $s \leq 0$ satisfying

$$
h(s)= \begin{cases}s & \text { for }-\varepsilon_{2} \leq s \leq 0 \\ \frac{1}{2}\left(\varepsilon_{1}+\varepsilon_{2}\right) & \text { for } s \leq-\varepsilon_{1}\end{cases}
$$

and

$$
h^{\prime}(s)>0 \text { for }-\varepsilon_{1}<s<-\varepsilon_{2}
$$

where $\varepsilon_{1}=\frac{1}{t}\left(1-e^{-1}\right)$ and $\varepsilon_{2}=\frac{1}{t}\left(1-e^{-1 / 2}\right)$. The function $\zeta:=h(\eta)$ is smooth in $\mathcal{D}$ and

$$
\nabla^{2} \zeta=h^{\prime} \nabla^{2} \eta+h^{\prime \prime} \nabla \eta \otimes \nabla \eta
$$

Since $h^{\prime} \geq 0$ and $h^{\prime \prime} \geq 0$ we have

$$
\lambda\left(\nabla^{2} \zeta\right) \in \overline{\Gamma_{k}} \text { in } \overline{\mathcal{D}} .
$$

Let $\rho \geq 0$ be a smooth cutoff function with compact support in $\mathcal{D}$ such that $\rho \equiv 1$ in the complement of $N_{\delta / 2}$. We consider the function

$$
v:=\zeta+c \rho w
$$

where $w$ is a smooth convex function satisfying

$$
\left(\operatorname{det} \nabla^{2} w\right)^{\frac{1}{n}} \geq 2 A \psi(1+|\nabla w|) \text { in } \mathcal{D},
$$

which can be constructed as in (3.6) with a suitable choice of $f$. Note that $v=0$ on $\partial \mathcal{D}$. Moreover,

$$
\nabla v=\nabla \zeta+c(\rho \nabla w+w \nabla \rho)
$$

and

$$
\nabla^{2} v=\nabla^{2} \zeta+c \rho \nabla^{2} w+c\left(\nabla \rho \otimes \nabla w+\nabla w \otimes \nabla \rho+w \nabla^{2} \rho\right) .
$$

Since $\zeta=\eta$ in $N_{\delta / 2}$, by (3.14) and (3.16) we may fix $c>0$ sufficiently small (which may depend on $A$, however) such that $\lambda\left(\nabla^{2} v\right) \in \Gamma_{k}$ in $N_{\delta / 2}$ and

$$
\sigma_{k}^{1 / k}\left(\nabla^{2} v\right) \geq \frac{1}{2} \sigma_{k}^{1 / k}\left(\nabla^{2} \zeta\right) \geq 4 A(1+|\nabla \eta|) \geq 2 A(1+|\nabla v|) \text { in } N_{\delta / 2}
$$

Since $\rho \equiv 1$ in the complement of $N_{\delta / 2}$ and $0 \leq h^{\prime} \leq 1$, we have

$$
|\nabla v| \leq|\nabla \zeta|+c|\nabla w|=h^{\prime}|\nabla \eta|+c|\nabla w| \text { in } \overline{\mathcal{D}} \backslash N_{\delta / 2}
$$


and

$$
\nabla^{2} v=\nabla^{2} \zeta+c \nabla^{2} w \in \Gamma_{k}, \text { in } \overline{\mathcal{D}} \backslash N_{\delta / 2} .
$$

By (3.17), (3.16), (3.18) and the concavity of $\sigma_{k}^{1 / k}$ in $\Gamma^{k}$,

$$
\begin{aligned}
\sigma_{k}^{1 / k}\left(\nabla^{2} v\right) & \geq \sigma_{k}^{1 / k}\left(h^{\prime} \nabla^{2} \eta+c \nabla^{2} w\right) \\
& \geq h^{\prime} \sigma_{k}^{1 / k}\left(\nabla^{2} \eta\right)+c \sigma_{k}^{1 / k}\left(\nabla^{2} w\right) \\
& \geq 8 A h^{\prime}(1+|\nabla \eta|)+2 A c(1+|\nabla w|) \\
& \geq 2 A(c+|\nabla v|) \quad \text { in } \overline{\mathcal{D}} \backslash N_{\delta / 2} .
\end{aligned}
$$

Here we have used the Newton-MacLaurin inequality

$$
\sigma_{k}^{1 / k}\left(\nabla^{2} w\right) \geq \sigma_{n}^{1 / n}\left(\nabla^{2} w\right)=\left(\operatorname{det}\left(\nabla^{2} w\right)\right)^{\frac{1}{n}}
$$

(Recall that $\sigma_{k}$ is normalized; see (3.13).) Finally, choosing $B$ sufficiently large we see from (3.20) and (3.21) that the function $\underline{u}:=B v+\varphi$ is admissible and satisfies (3.12).

Note that any admissible function in $\mathcal{D}$ is subharmonic and therefore satisfies the maximum principle. Since $\psi_{u} \geq 0$, taking

$$
A=\max _{x \in \overline{\mathcal{D}}} \psi(x, \bar{\varphi}), \quad \bar{\varphi}=\max _{\partial \mathcal{D}} \varphi
$$

in Lemma 3.4 we obtain an admissible subsolution for the Dirichlet problem (3.9), (3.11). Theorem 3.3 now follows from Theorem 1.3 in [6]. The proof of Theorem 3.3 is complete and therefore so is that of Theorem 1.2.

\section{REFERENCES}

[1] L. A. Caffarelli, L. Nirenberg and J. Spruck, The Dirichlet problem for nonlinear secondorder elliptic equations I. Monge-Ampère equations, Comm. Pure Applied Math. 37 (1984), 369-402.

[2] L. A. Caffarelli, L. Nirenberg and J. Spruck, The Dirichlet problem for nonlinear secondorder elliptic equations III: Functions of eigenvalues of the Hessians, Acta Math. 155 (1985), 261-301.

[3] E. Calabi, Examples of Bernstein problems for some nonlinear equations, Proc. Global Analysis, UC Berkeley, 1968.

[4] S.-Y. Cheng and S.-T. Yau, Maximal spacelike hypersurfaces in the Lorentz-Minkowski spaces, Annals of Math. 104(2) (1976), 407-419. 
[5] B. Guan, The Dirichlet problem for Monge-Ampère equations in non-convex domains and spacelike hypersurfaces of constant Gauss curvature, Trans. Amer. Math. Soc. 350 (1998), 4955-4971.

[6] B. Guan, The Dirichlet problem for Hessian equations on Riemannian manifolds, Calc. Var. PDE 8 (1999), 45-69.

[7] B. Guan and H.-Y, Jian The Monge-Ampère equation with infinite boundary value, Pacific J. Math. 216 (2004), 77-94.

[8] P.-F. Guan and Y.-Y. Li, The Weyl problem with nonnegative Gauss curvature, J. Differential Geom. 39 (1994), 331-342.

[9] Q. Han, On the isometric embedding of surfaces with Gauss curvature changing sign cleanly, Comm. Pure Appl. Math. 58 (2005), 285-295.

[10] Q. Han, Local isometric embedding of surfaces with Gauss curvature changing sign stably across a curve, Calc. Var. Partial Differential Equations 25 (2006), 79-103.

[11] Q. Han, J.-X. Hong and C.-S. Lin, Local isometric embedding of surfaces with nonpositive Gaussian curvature, J. Differential Geom. 63(2003), 475-520.

[12] J.-X. Hong, Realization in $\mathbb{R}^{3}$ of complete Riemannian manifolds with negative curvature, Comm. Anal. Geom. 1 (1993), 487-514.

[13] J.-X. Hong, Isometric embedding in $\mathbb{R}^{3}$ complete noncompact nonnegatively curved surfaces, Manuscripta Math. 94 (1997), 271-286.

[14] J.-X. Hong, Darboux equations and isometric embedding of Riemannian manifolds with nonnegative curvature in $\mathbb{R}^{3}$, Chinese Ann. Math. Ser. B 20 (1999), 123-136.

[15] J.-X. Hong, Boundary value problems for isometric embedding in $\mathbb{R}^{3}$ of surfaces, Partial Differential Equations and Their Applications (Wuhan, 1999), 87-98, World Sci. Publishing, River Edge, NJ, 1999.

[16] J.-X. Hong, Recent developments of realization of surfaces in $\mathbb{R}^{3}$, First International Congress of Chinese Mathematicians (Beijing, 1998), 47-62, AMS/IP Stud. Adv. Math. 20, Amer. Math. Soc., Providence, 2001.

[17] J.-X. Hong, Positive disks with prescribed mean curvature on the boundary, Asian J. Math. 5 (2001), 473-492.

[18] J.-X. Hong and C. Zuily, Isometric embedding of the 2-sphere with nonnegative curvature in $\mathbb{R}^{3}$, Math. Z. 219 (1995), 323-334.

[19] L. Nirenberg, The Weyl and Minkowski problems in differential geometry in the large, Comm. Pure Applied Math. 6 (1953), 337-394.

[20] C.-S. Lin, The local isometric embedding in $\mathbb{R}^{3}$ of 2-dimensional Riemannian manifolds with nonnegative curvature, J. Differential Geom. 21 (1985), 213-230.

[21] C.-S. Lin, The local isometric embedding in $\mathbb{R}^{3}$ of two-dimensional Riemannian manifolds with Gaussian curvature changing sign cleanly, Comm. Pure Appl. Math. 39 (1986), 867887.

[22] A. V. Pogorelov, Regularity of a convex surface with given Gaussian curvature, Mat. Sb. 31 (1952), 88-103.

[23] A. V. Pogorelov, Extrinsic Geometry of Convex Surfaces, Amer. Math. Soc., Providence, 1973. 
[24] R. M. Schoen and S.-T. Yau, Lectures on Differential Geometry, International Press, Cambridge, MA, 1994.

[25] S.-T. Yau, Open problems in geometry, Differential Geometry (Los Angeles, CA, 1990), 1-28, Proc. Sympos. Pure Math., 54 Part 1, Amer. Math. Soc., Providence, RI, 1993.

\section{Bo Guan}

Department of Mathematics, Ohio State University

Columbus, OH 43210, USA

E-mail: guan@math.osu.edu 